\title{
Alternative descriptions and bipartite compound quantum systems
}

\author{
G. Scolarici*and L. Solombrino ${ }^{\dagger}$ \\ Dipartimento di Fisica dell'Università del Salento and \\ INFN, Sezione di Lecce, I-73100 Lecce, Italy
}

November 1, 2018

\begin{abstract}
We analyze some features of alternative Hermitian and quasi-Hermitian quantum descriptions of simple and bipartite compound systems. We show that alternative descriptions of two interacting subsystems are possible if and only if the metric operator of the compound system can be obtained as tensor product of positive operators on component spaces. Some examples also show that such property could be strictly connected with symmetry properties of the non-Hermitian Hamiltonian.
\end{abstract}

\section{Introduction}

In the past years, since a conjecture due to Bender and Boettcher [1, a growing interest has been witnessed in PT-symmetric non-Hermitian Hamiltonians with real spectra [2, 3]. Today, it is well known that PT-symmetry actually constitutes a concrete, physically relevant realization of $\eta$-pseudo-Hermiticity property [4], [5] defined by relation

$$
\eta H \eta^{-1}=H^{\dagger},
$$

with $\eta$ Hermitian and invertible.

In the context of the pseudo-Hermitian quantum theory (PHQM) a relevant role is played by the quasi-Hermitian Hamiltonians, i. e., those pseudoHermitian Hamiltonians admitting a positive definite inner product invariant under their dynamics [6]. Although non-Hermitian, these Hamiltonians turn out to be sufficiently close to those of conventional quantum mechanics (QM) (in particular they are necessarily diagonalizable with real spectrum [5], 7])

\footnotetext{
*e-mail: scolarici@le.infn.it
}

†e-mail: solombrino@le.infn.it 
and therefore a standard quantum language is allowed to describe the predicted results.

Actually, a complete mathematical equivalence between PHQM and QM can be proven, at least if one considers simple quantum systems, since a unitary mapping exists which connects the corresponding Hilbert spaces [8, 9, 10]; more directly, such equivalence can also be proven by showing the equivalence of the spectra associated with the two systems [11] (we recall however that the physical interpretation of PHQM is still controversial, as the living debate on the quantum brachistochrone proves [12, [10], [13]). Finally, let us notice that these theories can be considered alternative Hamiltonian descriptions for quantum systems (see for instance [14 and references therein).

Now, a remarkable picture of Hermitian and quasi-Hermitian dynamics,

$$
i \frac{d}{d t}|\psi\rangle=H|\psi\rangle
$$

is the existence of a (possibly infinite) set of dynamically invariant $\eta$-inner product characterized by positive operators $\eta[4$ and defined by

$$
h_{\eta}(., .)=h_{1}(., \eta .),
$$

where $h_{1}(.,$.$) denotes the standard or fiducial inner product in the Hilbert space$ $\mathcal{H}$. Hence, the possibility of alternative quantum descriptions naturally arises in this context so that we will study in depth this topics both for Hermitian and for quasi-Hermitian Hamiltonians. In particular, in Sec. 2, we will show that the expectation value of the energy observable strongly depends on the alternative inner product, and that to different alternative descriptions associated with the same (non pure) density state, correspond different values of von Neumann entropy. These results can contribute, in our opinion, to clarify the real meaning of the above mentioned equivalence between PHQM and QM.

Moreover, since, of course, any physically meaningful theory must be able to describe compound systems, we will extend PHQM to include bipartite quantum systems, in order to verify if the equivalence between PHQM and QM (which, as we said above, has been already stated in literature only for simple systems) can be proven also at the level of compound systems. We recall that the problem to analyze to what extent alternative quantum descriptions survive when one considers compound systems and interactions among them was already raised on in Ref. [15] with respect to Hermitian dynamics. We will show in this paper that alternative descriptions play a crucial role in this respect; indeed, in Sec. 3, we prove a necessary and sufficient condition (propositions 2 and 3 ) which ensures such equivalence. In particular, we prove that quasi-Hermitian descriptions for bipartite compound quantum systems are permitted if and only if the positive operator which characterizes the alternative inner product can be written as the tensor product of two positive operators on the component spaces. (To avoid technicalities, we limit ourselves to consider here finite-dimensional bipartite systems.) As a consequence, severe restrictions arise about the equivalence between PHQM and QM for compound systems. 
Then, these general results are illustrated by some examples in Sec. 4, where also reduced density matrices via partial traces are introduced; in particular, the example in Subsec.4.3 shows a physical situation where alternative descriptions of two interacting subsystems associated with a quasi-Hermitian Hamiltonian are forbidden. Some concluding remarks are drawn in the last section.

\section{Alternative quasi-Hermitian descriptions}

We begin by discussing alternative descriptions for quantum systems in the case of quasi-Hermitian dynamics.

The spectral representation of a $\eta$-quasi-Hermitian Hamiltonian operator $H$ with a nondegenerate spectrum in terms of its biorthonormal eigenbasis, $\left\{\left|\psi_{n}\right\rangle,\left|\phi_{n}\right\rangle\right\}$, reads [5]

$$
H=\sum_{n} E_{n}\left|\psi_{n}\right\rangle\left\langle\phi_{n}\right|, \quad E_{n} \in \mathbb{R} .
$$

Furthermore,

$$
\eta=\sum_{n}\left|\phi_{n}\right\rangle\left\langle\phi_{n}\right|
$$

and

$$
\eta\left|\psi_{n}\right\rangle=\left|\phi_{n}\right\rangle .
$$

By using the inner product (3) and the spectral representation (4), the expectation value of $H$ in a (normalized) state,

$$
\frac{1}{\sqrt{\langle\psi|\eta| \psi\rangle}}|\psi\rangle=\frac{1}{\sqrt{\langle\psi|\eta| \psi\rangle}} \sum_{n}\left|\psi_{n}\right\rangle\left\langle\phi_{n} \mid \psi\right\rangle,
$$

can be computed:

$$
\begin{aligned}
h_{\eta}(\psi, H \psi) & \doteqdot \sum_{n} E_{n} p\left(E_{n}\right)=\sum_{n} E_{n} \frac{\left\langle\psi|\eta| \psi_{n}\right\rangle\left\langle\psi_{n}|\eta| \psi\right\rangle}{\langle\psi|\eta| \psi\rangle}= \\
& =\sum_{n} E_{n} \frac{\left\langle\psi_{n}|\eta| \psi\right\rangle\left\langle\psi|\eta| \psi_{n}\right\rangle}{\langle\psi|\eta| \psi\rangle}=\sum_{n} E_{n} \frac{\left\langle\phi_{n} \mid \psi\right\rangle\left\langle\psi|\eta| \psi_{n}\right\rangle}{\langle\psi|\eta| \psi\rangle}= \\
& =\sum_{n} \frac{\left\langle\phi_{n} \mid \psi\right\rangle\left\langle\psi|\eta H| \psi_{n}\right\rangle}{\langle\psi|\eta| \psi\rangle}=\operatorname{Tr}\left(\frac{|\psi\rangle\langle\psi| \eta}{\langle\psi|\eta| \psi\rangle} H\right)=\operatorname{Tr} \tilde{\rho} H
\end{aligned}
$$

where

$$
\tilde{\rho}=\frac{|\psi\rangle\langle\psi| \eta}{\langle\psi|\eta| \psi\rangle} .
$$

More generally, if $\rho$ denotes a generic (Hermitian, positive definite) density matrix $(\operatorname{Tr} \rho=1)$, we can associate with it a generalized density matrix $\tilde{\rho}$ by means of the one-to-one mapping in the following way [17, [10]: 


$$
\tilde{\rho}=\frac{\rho \eta}{\operatorname{Tr} \rho \eta}
$$

and obtain

$$
\langle H\rangle_{\eta}=\operatorname{Tr} \tilde{\rho} H .
$$

The dynamics of $\tilde{\rho}$ is ruled at infinitesimal level by the Liouville-von Neumann equation [17]

$$
\frac{d}{d t} \tilde{\rho}(t)=-i[H, \tilde{\rho}] .
$$

We note explicitly that the mapping (77) does not change the rank of the density matrices [18],

$$
\operatorname{rank} \tilde{\rho}=\operatorname{rank} \rho .
$$

Of course, in such scheme, to different metric operators $\eta$ and $\eta^{\prime}$, both fulfilling relation (11), correspond "alternative" descriptions of the same quantum system.

It is clear that changing the inner product corresponds to different expectation values of $H$ on the same state $|\psi\rangle$. In fact, from Eq. (6), we obtain in general,

$$
\langle H\rangle_{\eta}=\operatorname{Tr}\left(\frac{\rho \eta}{\operatorname{Tr} \rho \eta} H\right) \neq \operatorname{Tr}\left(\frac{\rho \eta^{\prime}}{\operatorname{Tr} \rho \eta^{\prime}} H\right)=\langle H\rangle_{\eta^{\prime}} .
$$

Moreover, denoting with $S(\widetilde{\rho})$ the von Neumann entropy associated with a density matrix $\widetilde{\rho}$, as a consequence of Eq. (10) we immediately get

$$
S(\widetilde{\rho})=-\operatorname{Tr}(\widetilde{\rho} \log \widetilde{\rho}) \neq-\operatorname{Tr}\left(\widetilde{\rho}^{\prime} \log \widetilde{\rho}^{\prime}\right)=S\left(\widetilde{\rho}^{\prime}\right),
$$

i. e., the von Neumann entropy depends on the alternative inner product. Then, we can conclude that the entropy and the expectation value of quasi-Hermitian observables strongly depend on the alternative description we consider on $\mathcal{H}$.

Note that if $\eta H \eta^{-1}=H^{\dagger}$ and $\eta^{\prime} H \eta^{-1}=H^{\dagger}$ with $\eta^{\prime} \neq \eta$, by performing in the Hilbert space $\mathcal{H}$ the linear transformation induced by $\eta^{\frac{1}{2}}$, we get

$$
H \rightarrow H^{\prime}=\eta^{\frac{1}{2}} H \eta^{-\frac{1}{2}}=H^{\prime \dagger},
$$

while the metric operators transform by congruence [19]:

$$
\begin{gathered}
\eta \rightarrow \eta^{-\frac{1}{2}} \eta(\eta)^{\dagger-\frac{1}{2}}=\eta^{-\frac{1}{2}} \eta \eta^{-\frac{1}{2}}=\mathbf{1}, \\
\eta^{\prime} \rightarrow \eta^{-\frac{1}{2}} \eta^{\prime} \eta^{-\frac{1}{2}}
\end{gathered}
$$

and the $\eta^{\prime}$-quasi-Hermiticity condition of $H$ implies

$$
\left[H^{\prime}, \eta^{-\frac{1}{2}} \eta^{\prime} \eta^{-\frac{1}{2}}\right]=0
$$

i. e., $\eta^{-\frac{1}{2}} \eta^{\prime} \eta^{-\frac{1}{2}}$ belongs to the commutant of $H^{\prime}$ and represents the operator which connect an alternative inner product invariant under time-translation 
generated by $H^{\prime}$ to the fiducial scalar product (see also [16]). Then, in the space where $H$ becomes Hermitian alternative inner products can be obtained by means of positive definite operators in the commutant of $H^{\prime}$ (coming back, the mapping (11), (12), can also be useful to compute the full set of $\eta$ operators fulfilling the quasi-Hermiticity condition). Moreover, the same calculations show that all the statements for quasi-Hermitian Hamiltonians also hold, with minimal and obvious changes, for the Hermitian ones.

\section{Quasi-Hermitian bipartite quantum systems}

Let us consider a compound bipartite quantum system which dynamics is described in a Hilbert space $\mathcal{H}^{n m}$ of dimension $n m$ by an evolution operator $U$ such that

$$
U^{\dagger} \eta U=\eta
$$

where

$$
U(t)=e^{-i H t}
$$

and the time-independent Hamiltonian $H$ satisfies,

$$
\eta H \eta^{-1}=H^{\dagger}, \quad \eta>0 .
$$

Then, the alternative Hermitian structure, $h_{\eta}(.,)=.h_{1}(., \eta$.$) , is invariant under$ the dynamics generated by $H$.

Now, a natural question arises: Is it possible a proper quantum mechanical description of such quasi-Hermitian compound quantum systems in terms of their corresponding component systems?

In order to answer to this question, as a preliminary step, we put the following proposition which gives a necessary condition for an operator $\eta$ of dimension $n m$ to be written as the tensor product of two operators of dimension $n$ and $m$ respectively: $\eta=\xi \otimes \zeta$.

Proposition 1. A positive Hermitian operator $\eta$ with eigenvalues $\left\{\eta_{i j}: i=\right.$ $1,2, \ldots, n ; j=1,2, \ldots, m\}$ acting on the complex vector space $\mathcal{H}^{n m}$ of dimension $n m$, can be decomposed as $\eta=\xi \otimes \zeta$ where $\xi$ and $\zeta$ represent positive Hermitian operators acting on $\mathcal{H}^{n}$ and $\mathcal{H}^{m}$ with eigenvalues $\left\{\xi_{i}: i=1,2, \ldots, n\right\}$ and $\left\{\zeta_{j}\right.$ : $j=1,2, \ldots, m\}$ respectively, only if the following $n^{2}$ and $m^{2}$ constraints are satisfied: $\frac{\eta_{i j}}{\xi_{i}}=\frac{\eta_{i^{\prime} j}}{\xi_{i^{\prime}}}, i, i^{\prime}=1,2, \ldots, n$ and $\frac{\eta_{i j}}{\zeta_{j}}=\frac{\eta_{i j^{\prime}}}{\zeta_{j^{\prime}}}, j, j^{\prime}=1,2, \ldots, m$.

Proof. Let us suppose $\eta=\xi \otimes \zeta$. Then, diagonalizing $\xi$ and $\zeta$ (and suitably ordering the spectra) we get

$$
\xi_{i} \zeta_{j}=\eta_{i j}
$$

where $i=1,2, \ldots, n, j=1,2, \ldots, m$. From the invertibility of $\xi$ and $\zeta$ we immediately get 


$$
\frac{\eta_{i j}}{\xi_{i}}=\frac{\eta_{i^{\prime} j}}{\xi_{i^{\prime}}}, i, i^{\prime}=1,2, \ldots, n \quad \text { or } \quad \frac{\eta_{i j}}{\zeta_{j}}=\frac{\eta_{i j^{\prime}}}{\zeta_{j^{\prime}}}, j, j^{\prime}=1,2, \ldots, m
$$

On the other hand, a sufficient condition in order that a given $\eta$ operator satisfying the previous constraints can be written as $\eta=\xi \otimes \zeta$, is that it must be diagonalizable by means of a unitary transformation of the form $U_{1} \otimes U_{2}$ where $U_{1} \in U(n, \mathbf{C})$ and $U_{2} \in U(m, \mathbf{C})$.

Now, we denote with

$$
U\left(n m, \mathbf{C}, h_{\eta}\right),
$$

the group which preserve the alternative Hermitian structure $h_{\eta}$.

Having in mind component systems, and recalling that in standard QM for any $U_{1} \in U(n, \mathbf{C})$ and $U_{2} \in U(m, \mathbf{C})$

$$
U_{1} \otimes U_{2} \in U(n m, \mathbf{C})
$$

the following proposition gives a necessary and sufficient condition for the Hilbert spaces $\mathcal{H}^{n}$ and $\mathcal{H}^{m}$ associated with the component systems to be provided of suitable alternative Hermitian structures $h_{\xi}$ and $h_{\zeta}$ such that for any $U_{\xi} \in$ $U\left(n, \mathbf{C}, h_{\xi}\right)$ and $U_{\zeta} \in U\left(m, \mathbf{C}, h_{\zeta}\right)$

$$
U_{\xi} \otimes U_{\zeta} \in U\left(n m, \mathbf{C}, h_{\eta}\right) .
$$

Proposition 2. For any $U_{\xi} \in U\left(n, \mathbf{C}, h_{\xi}\right)$ and $U_{\zeta} \in U\left(m, \mathbf{C}, h_{\zeta}\right)$, the group $U\left(n m, \mathbf{C}, h_{\eta}\right)$ contains the transformations

$$
U_{\xi} \otimes U_{\zeta}
$$

if and only if

$$
\eta=\xi \otimes \zeta .
$$

Denoting with $\mathfrak{H}_{\xi}^{n}, \mathfrak{H}_{\zeta}^{m}$ and $\mathfrak{H}_{\eta}^{n m}$ the group algebras associated with $U\left(n, \mathbf{C}, h_{\xi}\right)$, $U\left(m, \mathbf{C}, h_{\zeta}\right)$ and $U\left(n m, \mathbf{C}, h_{\eta}\right)$ respectively, proposition 2 can be equivalently restated in the following form:

Proposition 3. The set $\mathfrak{H}_{\eta}^{n m}$ of $\eta$-quasi-Hermitian matrices of dimension $n m$ contain as its subset

$$
K^{n m}=\left\{O_{\xi} \otimes O_{\zeta} \mid O_{\xi} \in \mathfrak{H}_{\xi}^{n}, O_{\zeta} \in \mathfrak{H}_{\zeta}^{m}\right\},
$$

where $\mathfrak{H}_{\xi}^{n}$ represent the set of $\xi$-quasi-Hermitian matrices of dimension $n$ and $\mathfrak{H}_{\zeta}^{m}$ represent the set of $\zeta$-quasi-Hermitian matrices of dimension $m$, if and only if

$$
\eta=\xi \otimes \zeta .
$$

Proof. Let us suppose $\eta=\xi \otimes \zeta$. Then, trivially, the set $K^{n m}$ is constituted by $\eta$-quasi-Hermitian matrices, hence, $K^{n m} \cap \mathfrak{H}_{\eta}^{n m} \equiv K^{n m}$. Conversely, let us 
suppose $\eta \neq \xi \otimes \zeta$ for any positive operator $\xi$ and $\zeta$. The set $K^{n m}$ is obviously an irreducible set of $\xi \otimes \zeta$-quasi-Hermitian matrices; then, by a known result in literature (see Ref. [6]), the metric is unique (up to a normalization factor), hence, it coincides with $\xi \otimes \zeta$. Then, the set $K^{n m}$ contain some matrices that cannot be $\eta$-quasi-Hermitians, hence, $K^{n m} \cap \mathfrak{H}_{\eta}^{n m} \supset K^{n m}$.

A direct consequence of proposition 3 is that the tensor product of two observables in $\left(\mathcal{H}^{n}, h_{\xi}\right)$ and $\left(\mathcal{H}^{m}, h_{\zeta}\right)$, is certainly an observable in $\left(\mathcal{H}^{n m}, h_{\eta}\right)$, if and only if $\eta=\xi \otimes \zeta$. In particular, let us assume, $\eta \neq \xi \otimes \zeta$. Then, some elements of the set $\left\{O_{\xi} \otimes \mathbf{1}^{m}, \mathbf{1}^{n} \otimes O_{\zeta}\right\}$, cannot be observable. In fact, let us suppose

$$
\eta\left(O_{\xi} \otimes \mathbf{1}^{m}\right) \eta^{-1}=O_{\xi}^{\dagger} \otimes \mathbf{1}^{m}
$$

and

$$
\eta\left(\mathbf{1}^{n} \otimes O_{\zeta}\right) \eta^{-1}=\mathbf{1}^{n} \otimes O_{\zeta}^{\dagger}
$$

for any $O_{\xi}$ and $O_{\zeta}$. From the commutativity,

$$
\left[O_{\xi} \otimes \mathbf{1}^{m}, \mathbf{1}^{n} \otimes O_{\zeta}\right]=0
$$

we immediately get

$$
\eta\left(O_{\xi} \otimes O_{\zeta}\right) \eta^{-1}=O_{\xi}^{\dagger} \otimes O_{\zeta}^{\dagger}
$$

whereas, according with the above hypothesis,

$$
(\xi \otimes \zeta)\left(O_{\xi} \otimes O_{\zeta}\right)(\xi \otimes \zeta)^{-1}=O_{\xi}^{\dagger} \otimes O_{\zeta}^{\dagger} .
$$

From the irreducibility of the set $\left\{O_{\xi} \otimes O_{\zeta}\right\}$, (see proposition 3 ) the thesis follows at once.

Then we can conclude that any $\eta$-quasi-Hermitian compound quantum system admits a proper quantum mechanical description in terms of component systems if and only if $\eta=\xi \otimes \zeta$.

Note, of course, that if an operator $\eta$ admits a decomposition $\eta=\xi \otimes \zeta$, such decomposition is not unique. In fact, we can for instance change the operators $\xi$ and $\zeta$ by multiplying them by (positive) factors $r$ and $\frac{1}{r}$ respectively.

Remark. Clearly if $\eta=\xi \otimes \zeta$ the peculiarity of a state, associated with a compound system, to be entangled or not does not depend on the alternative structures $h_{\xi}$ and $h_{\zeta}$ on component spaces. In fact, let us consider

$$
|\beta\rangle=|\chi\rangle \otimes|\omega\rangle \in \mathcal{H}^{n m}
$$

Then,

$$
\left|\beta^{\prime}\right\rangle=S|\beta\rangle
$$

is entangled if and only if $S \in G L(n m, \mathbb{C})$ and $S \neq S_{1} \otimes S_{2}$ for any $S_{1} \in G L(n, \mathbb{C})$ and $S_{2} \in G L(m, \mathbb{C})$. Hence, the (alternative) Hermitian structure does not play here any role. 


\section{Examples}

We illustrate the general results in the previous sections by means of some examples. In order to do that, we first introduce the reduced density matrices for bipartite quasi-Hermitian systems via partial trace operation.

Let be given a $\eta$-quasi-Hermitian Hamiltonian associated with a bipartite system,

$$
H=H_{A} \otimes \mathbf{1}^{m}+\mathbf{1}^{n} \otimes H_{B}+V_{i n t}
$$

where we assume for the sake of simplicity that

$$
H_{A}=\sum_{i=1}^{n} a_{i}\left|\psi_{i}\right\rangle\left\langle\phi_{i}\right|, \quad a_{i} \in \mathbb{R}
$$

and

$$
H_{B}=\sum_{j=1}^{m} b_{j}\left|\Psi_{j}\right\rangle\left\langle\Phi_{j}\right|, \quad b_{j} \in \mathbb{R}
$$

are quasi-Hermitians with a nondegenerate spectrum. Then, the more general $\xi$ and $\zeta$ operators satisfying the quasi-Hermiticity conditions, $\xi H_{A} \xi^{-1}=H_{A}^{\dagger}$ and $\zeta H_{B} \zeta^{-1}=H_{B}^{\dagger}$, are respectively given by

$$
\xi=\sum_{i=1}^{n} r_{i}\left|\phi_{i}\right\rangle\left\langle\phi_{i}\right|, \quad r_{i}>0
$$

and

$$
\zeta=\sum_{j=1}^{m} s_{j}\left|\Phi_{j}\right\rangle\left\langle\Phi_{j}\right|, \quad s_{j}>0 .
$$

Then, any state $\frac{|\alpha\rangle}{\langle\alpha|\eta| \alpha\rangle}$ in the space $\mathcal{H}^{n m}$ provided with Hermitian structure $h_{\eta}$, can be decomposed on the biorthonormal basis $\left\{\left|\psi_{i}\right\rangle \otimes\left|\Psi_{j}\right\rangle,\left|\phi_{i}\right\rangle \otimes\left|\Phi_{j}\right\rangle\right\}$ :

$$
\begin{aligned}
& \frac{1}{\langle\alpha|\eta| \alpha\rangle}|\alpha\rangle \\
= & \left(\sum_{i, j}\left|\psi_{i}\right\rangle \otimes\left|\Psi_{j}\right\rangle\left(\left\langle\Phi_{j}\right| \otimes\left\langle\phi_{i}\right|\right)\right) \frac{1}{\langle\alpha|\eta| \alpha\rangle}|\alpha\rangle,
\end{aligned}
$$

and the associated rank-one density matrix reads

$$
\widetilde{\rho}^{A B}=\frac{|\alpha\rangle\langle\alpha| \eta}{\langle\alpha|\eta| \alpha\rangle} .
$$

Then, we immediately get 


$$
\widetilde{\rho}_{A}=\operatorname{Tr}_{B} \widetilde{\rho}^{A B}=\sum_{j=1}^{m}\left\langle\Phi_{j}\left|\widetilde{\rho}^{A B}\right| \Psi_{j}\right\rangle
$$

and

$$
\tilde{\rho}_{B}=\operatorname{Tr}_{A} \widetilde{\rho}^{A B}=\sum_{i=1}^{n}\left\langle\phi_{i}\left|\widetilde{\rho}^{A B}\right| \psi_{i}\right\rangle .
$$

Moreover, being $\eta=\xi \otimes \zeta$, we obtain

$$
\widetilde{\rho}_{A}=\operatorname{Tr}_{B} \widetilde{\rho}^{A B}=\frac{\rho^{A} \xi}{\operatorname{Tr} \rho^{A} \xi} \quad \text { and } \quad \widetilde{\rho}_{B}=\operatorname{Tr}_{A} \widetilde{\rho}^{A B}=\frac{\rho^{B} \zeta}{\operatorname{Tr} \rho^{B} \zeta},
$$

where $\rho^{A}$ and $\rho^{B}$ denote the partial traces associated with the state $\frac{|\alpha\rangle\langle\alpha|}{\langle\alpha \mid \alpha\rangle}$ in the fiducial (standard) description.

Now, we will consider some examples. In the first one, a dynamics generated by an Hermitian Hamiltonian associated with a composite system on a four dimensional Hilbert space is described in terms of two alternative invariant inner products. In the second one, alternative descriptions of a $P T$-symmetric quasi-Hermitian dynamics recently introduced in literature are considered. In the third one, alternative descriptions of a quasi-Hermitian not $P T$-symmetric dynamics are considered.

\subsection{An Hermitian Hamiltonian}

Because of the physical relevance of two qubit quantum gates, we shall now consider alternative descriptions for an optimal entanglement generation recently introduced in literature [20]. The system we consider is composed of two qubits $A$ and $B$, hence $\mathcal{H} \equiv \mathbb{C}^{4}$.

The Hamiltonian and the evolution operator of the overall system are

$$
\begin{aligned}
H= & \sigma_{3}^{A} \otimes \mathbf{1}^{B}+\mathbf{1}^{A} \otimes \sigma_{3}^{B}+V_{i n t}= \\
& \left(\begin{array}{cccc}
1 & 0 & 0 & 0 \\
0 & -1 & 0 & 0 \\
0 & 0 & -1 & 0 \\
0 & 0 & 0 & 1
\end{array}\right), \\
U= & \cos t \mathbf{1}^{A} \otimes \mathbf{1}^{B}-i \sin t \sigma_{3}^{A} \otimes \sigma_{3}^{B}= \\
& \left(\begin{array}{cccc}
e^{-i t} & 0 & 0 & 0 \\
0 & e^{i t} & 0 & 0 \\
0 & 0 & e^{i t} & 0 \\
0 & 0 & 0 & e^{-i t}
\end{array}\right) .
\end{aligned}
$$

Let the initial state be 


$$
\rho^{A}(0) \otimes \rho^{B}(0)=\frac{1}{2}\left(\begin{array}{cc}
1 & 1 \\
1 & 1
\end{array}\right) \otimes \frac{1}{2}\left(\begin{array}{cc}
1 & -i \\
i & 1
\end{array}\right) .
$$

At time $t$ we get

$$
\rho^{A B}(t)=U(t) \rho^{A B}(0) U(t)^{\dagger}
$$

and we obtain by partial traces the final states

$$
\begin{aligned}
& \rho^{A}(t)=\operatorname{Tr}_{B} \rho^{A B}=\frac{1}{2}\left(\begin{array}{cc}
1 & \cos 2 t \\
\cos 2 t & 1
\end{array}\right), \\
& \rho^{B}(t)=\operatorname{Tr}_{A} \rho^{A B}=\frac{1}{2}\left(\begin{array}{cc}
1 & -i \cos 2 t \\
i \cos 2 t & 1
\end{array}\right) .
\end{aligned}
$$

We stress that at the time $t_{\text {bell }}=\pi / 4$, the overall state $\rho^{A B}\left(t=t_{\text {bell }}\right)$ is equivalent to a Bell state $\frac{1}{\sqrt{2}}(|00\rangle+|11\rangle)$.

The von Neumann entropy gives an entanglement measure:

$$
\begin{aligned}
S\left(\rho^{A}(t)\right)= & -\operatorname{Tr}\left(\rho^{A} \log \rho^{A}\right)= \\
& -\left(\sin ^{2} t\right) \log \left(\sin ^{2} t\right)-\left(\cos ^{2} t\right) \log \left(\cos ^{2} t\right) .
\end{aligned}
$$

In particular, $S\left(\rho^{A}(t)\right)=0$ when the state $\rho^{A B}(t)$ becomes separable and this happens when the purity [20] of both the reduced density matrices,

$$
\begin{aligned}
& P_{\rho^{A}(t)}=\operatorname{Tr} \rho^{A}(t)^{2}=\frac{1}{2}\left(1+(\cos 2 t)^{2}\right), \\
& P_{\rho^{B}(t)}=\operatorname{Tr} \rho^{B}(t)^{2}=\frac{1}{2}\left(1+(\cos 2 t)^{2}\right),
\end{aligned}
$$

becomes 1 , that is when $t=\frac{k \pi}{2}, k \in \mathbb{N}$.

Now, let us consider in the Hilbert space associated with the compound system the most general (in the sense of proposition 2) alternative scalar product which is connected with the fiducial one by means of the (positive) operator,

$$
\eta=\xi \otimes \zeta=\left(\begin{array}{cc}
\xi_{1} & 0 \\
0 & \xi_{2}
\end{array}\right) \otimes\left(\begin{array}{cc}
\zeta_{1} & 0 \\
0 & \zeta_{2}
\end{array}\right)
$$

The Hermitian structures $h_{\eta}$ and $h_{\xi}, h_{\zeta}$ are well defined alternative inner products for composite and component systems respectively. In fact,

$$
\begin{aligned}
& {[H, \eta]=0, \quad U^{\dagger} \eta U=\eta,} \\
& {\left[\sigma_{3}^{A}, \xi\right]=0, \quad\left[\sigma_{3}^{B}, \zeta\right]=0,}
\end{aligned}
$$

hence, $H, \sigma_{3}^{A}$ and $\sigma_{3}^{B}$ are also Hermitian with respect to $h_{\eta}, h_{\xi}$ and $h_{\zeta}$, respectively. 
The initial density matrix in the description associated with $h_{\eta}$ reads

$$
\widetilde{\rho}^{A B}(0)=\frac{\left(\rho^{A}(0) \otimes \rho^{B}(0)\right) \eta}{\operatorname{Tr}\left(\rho^{A}(0) \otimes \rho^{B}(0)\right) \eta} .
$$

At time $t$ we get

$$
\widetilde{\rho}^{A B}(t)=U(t) \widetilde{\rho}^{A B}(0) U(t)^{\dagger}
$$

and from Eq. (20), the reduced density matrices can be computed:

$$
\begin{aligned}
\widetilde{\rho}^{A}(t)= & \operatorname{Tr}_{B} \widetilde{\rho}^{A B}= \\
& \frac{1}{\xi_{1}+\xi_{2}}\left(\begin{array}{cc}
\xi_{1} & \xi_{2} \cos 2 t \\
\xi_{1} \cos 2 t & \xi_{2}
\end{array}\right), \\
\widetilde{\rho}^{B}(t)= & \operatorname{Tr}_{A} \widetilde{\rho}^{A B}= \\
& \frac{1}{\zeta_{1}+\zeta_{2}}\left(\begin{array}{cc}
\zeta_{1} & -i \zeta_{2} \cos 2 t \\
i \zeta_{1} \cos 2 t & \zeta_{2}
\end{array}\right) .
\end{aligned}
$$

Note that $\widetilde{\rho}^{A}(t)$ and $\tilde{\rho}^{B}(t)$ are $\xi$ - and $\zeta$-quasi-Hermitian respectively.

The eigenvalues of $\widetilde{\rho}^{A}(t)$ are:

$$
r_{ \pm}=\frac{1}{2}\left(1 \pm \frac{\sqrt{\xi_{1}^{2}+\xi_{2}^{2}+2 \xi_{1} \xi_{2} \cos 4 t}}{\xi_{1}+\xi_{2}}\right)
$$

hence, its von Neumann entropy reads now

$$
\begin{aligned}
S\left(\widetilde{\rho}^{A}(t)\right)= & -\operatorname{Tr}\left(\widetilde{\rho}^{A} \log \widetilde{\rho}^{A}\right)= \\
& -r_{+} \log r_{+}-r_{-} \log r_{-} .
\end{aligned}
$$

It is then evident that, the entropy depends on the alternative scalar product, in fact, from Eqs. (23), (26), we immediately get

$$
S\left(\rho^{A}(t)\right) \neq S\left(\widetilde{\rho}^{A}(t)\right) .
$$

Then, the entanglement measure strongly depends on the alternative Hermitian structure.

It is worthwhile to note however that, $S\left(\widetilde{\rho}^{A}(t)\right)=0$ when the purity of both the reduced density matrices

$$
\begin{aligned}
& P_{\widetilde{\rho}^{A}(t)}=\operatorname{Tr} \widetilde{\rho}^{A}(t)^{2}=\frac{1}{2}\left(1+\frac{\xi_{1}^{2}+\xi_{2}^{2}+2 \xi_{1} \xi_{2} \cos 4 t}{\left(\xi_{1}+\xi_{2}\right)^{2}}\right), \\
& P_{\widetilde{\rho}^{B}(t)}=\operatorname{Tr} \widetilde{\rho}^{B}(t)^{2}=\frac{1}{2}\left(1+\frac{\zeta_{1}^{2}+\zeta_{2}^{2}+2 \zeta_{1} \zeta_{2} \cos 4 t}{\left(\zeta_{1}+\zeta_{2}\right)^{2}}\right),
\end{aligned}
$$


becomes 1 and this happens again when $t=\frac{k \pi}{2}, k \in \mathbb{N}$.

From Eqs. (24), (27) we conclude that whereas the entropy depends on the alternative inner product, the peculiarity of a state to be entangled or not does not depend on the alternative description (see also Eq. (9)) as we can expect from general considerations (see the remark in section 3 ).

\subsection{A $P T$-symmetric Hamiltonian}

Now, we discuss a recently introduced coupling between two qubits separately described by a Hermitian Hamiltonian and by a $P T$-symmetric Hamiltonian respectively [21, in terms of compound and component systems. In particular, we consider the Hermitian Hamiltonian

$$
H_{A}=\left(\begin{array}{cc}
1 & 0 \\
0 & 1
\end{array}\right)
$$

and the non-Hermitian Hamiltonian with real eigenvalues

$$
H_{B}=\frac{1}{2}\left(\begin{array}{cc}
\sqrt{3}+i & 2 \\
2 & \sqrt{3}-i
\end{array}\right)
$$

both the above Hamiltonians are $P T$-symmetric, where

$$
P=\sigma_{1}=\left(\begin{array}{cc}
0 & 1 \\
1 & 0
\end{array}\right), \quad T=K,
$$

( $K$ denotes complex conjugation). Then, we couple them by means of nonzero elements in the off-diagonal sectors, obtaining so (with a coupling constant $\left.\epsilon=\frac{1}{2}\right)$ :

$$
\begin{aligned}
& H=H_{A} \otimes \mathbf{1}^{B}+\mathbf{1}^{A} \otimes H_{B}+V_{\text {int }}= \\
& =\frac{1}{2}\left(\begin{array}{cccc}
2 & 0 & 1 & 0 \\
0 & 2 & 0 & 1 \\
1 & 0 & \sqrt{3}+i & 2 \\
0 & 1 & 2 & \sqrt{3}-i
\end{array}\right) .
\end{aligned}
$$

As we said above, such Hamiltonian is obtained by a suitable choice of parameters from the one considered in [21].

The coupling terms are chosen in such a way that $H$ remains invariant under the combined parity reflection and time reversal, where $T=K$ and

$$
P=\mathbf{1}^{A} \otimes \sigma_{1}=\left(\begin{array}{cccc}
0 & 1 & 0 & 0 \\
1 & 0 & 0 & 0 \\
0 & 0 & 0 & 1 \\
0 & 0 & 1 & 0
\end{array}\right)
$$

Being $\epsilon=\frac{1}{2}$, the eigenvalues of the combined system are real [21] and a positive definite $\eta$ can be written as 


$$
\eta=\mathbf{1}^{A} \otimes \zeta
$$

where $\zeta H_{B} \zeta^{-1}=H_{B}^{\dagger} ;$ for instance,

$$
\zeta=\left(\begin{array}{cc}
2 & -\sqrt{2}-i \\
-\sqrt{2}+i & 2
\end{array}\right)
$$

Clearly, by considering the (infinite) set of positive operators $\zeta$ satisfying the quasi-Hermiticity condition, $\zeta H_{B} \zeta^{-1}=H_{B}^{\dagger}$, we obtain a set of possible $\eta$ operators on $\mathbb{C}^{4}$ of the form, $\eta=\xi \otimes \zeta$, permitting alternative quasi-Hermitian descriptions of our system in terms of its component systems. For the sake of brevity we do not compute here the evolution of the overall system, the reduced density matrices and their von Neumann entropy as in the previous example.

\subsection{A quasi-Hermitian not $P T$-symmetric Hamiltonian}

Finally, let us consider a quasi-Hermitian Hamiltonan $H$ on $\mathbb{C}^{4}$, obtained by taking the direct sum of the Hermitian not PT-symmetric Hamiltonian, $H_{A}=$ $\left(\begin{array}{ll}1 & 0 \\ 0 & 2\end{array}\right)$, and the $P T$-symmetric Hamiltonian, $H_{B}$ given in Eq. (29):

$$
H=\left(\begin{array}{cc}
H_{A} & 0 \\
0 & H_{B}
\end{array}\right)=\frac{1}{2}\left(\begin{array}{cccc}
2 & 0 & 0 & 0 \\
0 & 4 & 0 & 0 \\
0 & 0 & \sqrt{3}+i & 2 \\
0 & 0 & 2 & \sqrt{3}-i
\end{array}\right)
$$

A direct computation shows that $H$ is not invariant under the combined parity reflection given by (31) and time-reversal $T=K$, but it is surely quasiHermitian since it is diagonalizable with real spectrum.

Let us show that the Hamiltonian (33) cannot admit a positive $\eta$ operator, satisfying the quasi-Hermiticity condition, of the form $\eta=\xi \otimes \zeta$, were $\xi$ and $\zeta$ are positive operators on $\mathbb{C}^{2}$. In fact, writing

$$
\xi=\left(\begin{array}{cc}
a & z \\
z^{*} & b
\end{array}\right), \quad a, b \in \mathbb{R}, \quad z \in \mathbb{C},
$$

the conditions: $\operatorname{det} \xi>0(\Rightarrow a b>0)$ and $\operatorname{Tr} \xi=a+b>0$, which ensure the positivity of $\xi$, imply that $a, b$ must be non-zero, positive real numbers.

Let us now consider the Kronecker product

$$
\eta=\xi \otimes \zeta=\left(\begin{array}{cc}
a \zeta & z \zeta \\
z^{*} \zeta & b \zeta
\end{array}\right)
$$

and impose

$$
\eta H=H^{\dagger} \eta .
$$

Eq. (34) is equivalent to the following matrix equations: 


$$
\begin{gathered}
\zeta H_{A}=H_{A} \zeta, \\
\zeta H_{B}=H_{B}^{\dagger} \zeta, \\
z\left(\zeta H_{B}-H_{A} \zeta\right)=0 .
\end{gathered}
$$

From Eq. (35) we immediately obtain that $\zeta$ must have a diagonal form, but a direct computation shows that no diagonal $\zeta$ can satisfy Eq. (36). Then, in this case no invertible, Hermitian positive operator $\eta$ exists which satisfies the condition $\eta=\xi \otimes \zeta$.

Then, we conclude that in this case, quasi-Hermitian descriptions for subsystems are forbidden.

\section{Concluding remarks}

In this paper we considered some features of alternative descriptions of simple and compound quantum systems and we have shown, also by means of examples, that the entanglement measure (von Neumann entropy) strongly depends on the alternative Hermitian structure. Moreover, we have analyzed to what extent Hermitian and quasi-Hermitian quantum descriptions of compound systems survive.

The main result of our paper is that if (and only if) the alternative Hermitian structure is connected with the fiducial ones by means of a positive operator $\eta$ such that

$$
\eta=\xi \otimes \zeta
$$

the projection on the component spaces can be performed via partial trace operation.

On the contrary, if

$$
\eta \neq \xi \otimes \zeta
$$

quasi-Hermitian descriptions for the component subsystems cannot be obtained and the corresponding physical theories are inconsistent (at least, according with the usual physical interpretation of the mathematical entities).

These results, as a consequence, pose severe restrictions on PHQM, in particular with respect to the asserted equivalence between such theories and standard Quantum Mechanics.

In fact, we observe that whereas the set of alternative inner product associated with Hermitian Hamiltonians admits a not void subset of operators of the form $\eta=\xi \otimes \zeta$ (in fact the identity trivially belongs to this subset), the example in Subsec.4.3 shows that when $\eta$-quasi-Hermitian Hamiltonians are considered the existence of a such form of $\eta$ cannot be assured. However, the example in 
Subsec.4.2 suggests that the existence of operators of the form $\eta=\xi \otimes \zeta$ could be ensured in case of $P T$-symmetric Hamiltonians.

A complete characterization of the subclass of quasi-Hermitian Hamiltonians admitting $\eta$ operators of the form $\eta=\xi \otimes \zeta$ and the generalization of these results to multipartite quantum systems will be considered in a forthcoming paper [22].

We hope that the present developments on alternative descriptions for quasiHermitian dynamics associated with bipartite compound quantum systems could be also useful, as a preliminary step, in order to study the entanglement in the context of formulations of quantum mechanics with non-Hermitian opera-

tors and to obtain a classification of (positive) dynamical maps in the space of quasi-Hermitian density matrices.

Acknowledgements The authors wishes to thank professor H. F. Jones and one of the anonymous referees for their suggestions about an erroneous example in a previous version of the present paper.

\section{References}

[1] C. M. Bender and S. Boettcher, Phys. Rev. Lett., 80, 5243 (1998).

[2] Proceedings of the Ist, IInd, IIIrd, IVth., Vth. and VIth. International Workshops on "Pseudo-Hermitian Hamiltonians in Quantum Physics" in Czech. J. Phys. 54 (2004), Nos. 1, 10, Czech. J. Phys. 55 (2005), J. Phys. A 39 (2006), Czech. J. Phys. 56 (2006) and J. Phys. A 41 (2008), respectively.

[3] C. M. Bender, D. C. Brody and H. F. Jones, Phys. Rev. Lett., 89, 270401 (2002).

[4] A. Mostafazadeh, J. Math. Phys., 43, 205 (2002).

[5] A. Mostafazadeh, J. Math. Phys., 43, 2814 (2002).

[6] F. G. Sholtz, H. B. Geyer and F. J. W. Hahne, Ann. Phys., 213, 74 (1992).

[7] G. Scolarici and L. Solombrino, J. Math. Phys., 44, 4450 (2003).

[8] A. Mostafazadeh, J. Phys. A, 36, 7081 (2003).

[9] A. Mostafazadeh and A. Batal, J. Phys. A, 37, 11645 (2004).

[10] A. Mostafazadeh, Phys. Rev. Lett., 99, 130502 (2007).

[11] H. F. Jones, J. Phys. A, 38, 1741 (2005).

[12] C. M. Bender, D. C. Brody and H. F. Jones, and B. K. Meister, Phys. Rev. Lett., 98, 040403 (2007).

[13] C. M. Bender, D. C. Brody and H. F. Jones, and B. K. Meister, "Comment on the Quantum Brachistochrone Problem" arXiv: quant-ph/08043487. 
[14] G. Marmo, G. Scolarici, A. Simoni and F. Ventriglia, Theor. Math. Phys. 144, 1190 (2005).

[15] G. Marmo, G. Morandi, A. Simoni and F. Ventriglia, J. Phys. A 35, 8393 (2002).

[16] A. Mostafazadeh, J. Phys. A, 41, 055304 (2008).

[17] G. Scolarici and L. Solombrino, Czech. J. Phys., 56, 935(2006).

[18] R. A. Horn and C. R. Johnson, Matrix Analysis v. 1 (Cambridge University Press, Cambridge, 1985).

[19] A. Blasi, G. Scolarici and L. Solombrino, J. Phys. A., 37, 4335 (2004).

[20] C. A. Rodriguez, A. Shaji and E. C. G. Sudarshan "Dynamics of Two Qubits: Decoherence and Entanglement Optimization Protocol" arXiv: quant-ph/0504051.

[21] C. M. Bender, H. F. Jones, J. Phys. A, 41, 244006 (2008).

[22] F. Masillo, G. Scolarici and L. Solombrino "Alternative descriptions and multibipartite compound quantum systems" in preparation. 